\title{
Automatic Detection of Seizure ONSET in PEDIATRIC EEG
}

\author{
Yusuf U Khan ${ }^{1}$, Omar Farooq ${ }^{2}$ and Priyanka Sharma ${ }^{2}$ \\ ${ }^{1}$ Electrical Department, Aligarh Muslim University, Aligarh \\ yusufkhan. ee@amu.ac.in \\ ${ }^{2}$ Electronics Department, Aligarh Muslim University, Aligarh \\ omarfarooq70@gmail.com, priya.32dec@gmail.com
}

\begin{abstract}
This paper proposes a method for automatic detection of seizure onset. Two statistical features: skewness and kurtosis with a wavelet based feature: normalized coefficient of variation (NCOV) were extracted from the data. The classification between normal and seizure EEGs was performed using simple linear classifier. The performance of the algorithm was tested on the 10 patient's data of CHB-MIT scalp EEG database. The data consisted of 55 seizures of 10646 seconds duration. The results show a mean latency of 3.2 seconds, a mean false detection rate of 1.1 false detections per hour and $100 \%$ sensitivity.
\end{abstract}

\section{KEYWORDS}

EEG, Latency, Skewness, Kurtosis, Normalized coefficient of variation (NCOV).

\section{INTRODUCTION}

Epilepsy, a second most common neurological disorder, is characterized by recurrent seizures. These seizures are the result of sudden excessive electric discharge in the human brain. It may occur in the brain locally called as partial seizures or involve the whole brain called as generalized seizures. Patients are often unaware of the occurrence of seizures which may increase the risk of physical injury. Studies show that about 50 million people worldwide have been suffering from this disease [1]. For the treatment of epilepsy, patients take antiepileptic drugs (AEDs) on a daily basis but unfortunately despite treatment about $25 \%$ of the patients continue to experience frequent seizures [2]. These patients suffer from the epilepsy that does not respond to AED and called as refractory epilepsy. Surgery is the most effective and generally adopted treatment for these patients, but can be done only when epileptogenic focus is identified accurately. For this purpose different type of tracers are employed as soon as possible after onset detection. Early detection of seizure onset would be helpful in the rapid injection of tracer and hence accurate localization of epileptogenic focus.

EEG has been an important clinical tool for the analysis and treatment of epilepsy [3]. The EEG is a multichannel recording that reflect the activity generated by number of neurons within the brain. It is generally recorded using the electrodes placed on the scalp. Visual inspection of the EEG data is done by specialists to analyze epilepsy. But observing EEG continuously for a long time is a very tedious task, since EEG data recordings create lengthy data [4]. Hence automatic seizure detection is essential in clinical practice

DOI : 10.5121/ijesa.2012.2309 
International Journal of Embedded Systems and Applications (IJESA) Vol.2, No.3, September 2012

Automatic detection of seizures through the analysis of scalp EEG has been an important area of research for the last few decades [5-14]. In 1976, Gotman and Gloor [5] proposed a method of recognition and quantification of interictal epileptic activity (spikes and sharp waves) in human scalp EEG. To perform the automatic recognition, the EEG of each channel was broken down into half waves. A wave was characterized by the durations and amplitudes of its two component half waves, by the second derivative at its apex measured relative to the background activity, and by the duration and amplitude of the following half wave. This method gave a good basis to the work in the field of seizure detection. The main limitation of the method was the absence of precise definition for an interictal epileptic event. In 1982, Gotman [6] proposed an improved method for automatic detection of seizures in EEG. After this many methods have been proposed to detect the seizures, but few of those were on onset detection of seizures.

Qu and Gotman [7] proposed a patient specific seizure onset detection method and achieved a sensitivity of $100 \%$ with mean latency of 9.4 seconds. The average false detections declared were 0.02 per hour. The algorithm was tested on 47 seizures of 12 patients. The drawback of this method was the need of template for the detection of seizures. In 2004, Gotman and Saab [8] designed an onset detection system. When it was tested using scalp EEG of 16 patients having 69 seizures, sensitivity of $77.9 \%$ with false detection rate of 0.9 per hour and median detection delay of 9.8 seconds were reported. Sorensen et al [2] used matching pursuit algorithm and achieved $78-100 \%$ sensitivity with 5-18 seconds delay in seizure onset detection while at the same time 0.2- 5.3 false positives per hour were declared. The method was evaluated using both scalp and intracranial EEG. Shoeb and Guttag [9] reported 96\% sensitivity and mean detection delay of 4.6 seconds when worked on CHB-MIT database [10]. In 2011, Kharbouch et al [11] proposed a method for seizure detection from iEEG. The data of 10 patients was utilized to extract both temporal and spectral features. The method detected $97 \%$ of 67 test seizures with a median detection delay of 5 seconds and a median false detection rate of 0.6 per 24 hour.

In this paper, a method to study the latency of seizure detection using two statistical features and a wavelet based feature has been proposed. Daubechies wavelet has been widely used for the seizure detection in EEG [7, 12,13]. The proposed algorithm uses the Daubechies wavelet (of order 4) to detect the onset of seizures present in the database.

\section{EXPERIMENTAL WORK}

\subsection{Database}

The database used in this study was CHB-MIT scalp EEG database which is freely available online [10]. It was collected at the Children's Hospital Boston and consists of EEG recordings from pediatric subjects, suffering from intractable seizures. Recordings, grouped into 24 cases, were collected from 23 subjects (5 males, ages 3-22, 17 females, ages 1.5-19 and 1 unknown).

All EEG signals were sampled at $256 \mathrm{~Hz}$ with 16-bit resolution. Most files contain 23 EEG channels (24 or 26 in a few cases). EEG data was recorded according to the standard 10-20 system. Overall this 24 patient dataset consisted of 916 hours of continuously recorded EEG and 198 seizures. First 10 patient's EEG from this database was used for this study. The line frequency of $60 \mathrm{~Hz}$ was removed from the database.

\subsection{Feature Extraction}

Feature extraction is a crucial step of seizure detection in which features of the data are investigated that is able to differentiate between the seizure and normal EEG data. Figure 1 show the histograms for randomly selected channel of EEG recorded in both cases: normal and seizure 
International Journal of Embedded Systems and Applications (IJESA) Vol.2, No.3, September 2012

of one of the patient present in the database. The observable differences in the dispersion and symmetry motivate the selection of higher-order statistics for the characterization of the EEG. In this study three features: NCOV (ratio of variance $(\sigma 2)$ and absolute mean $(\mu \mathrm{a})$ ), kurtosis and skewness were extracted from the data.

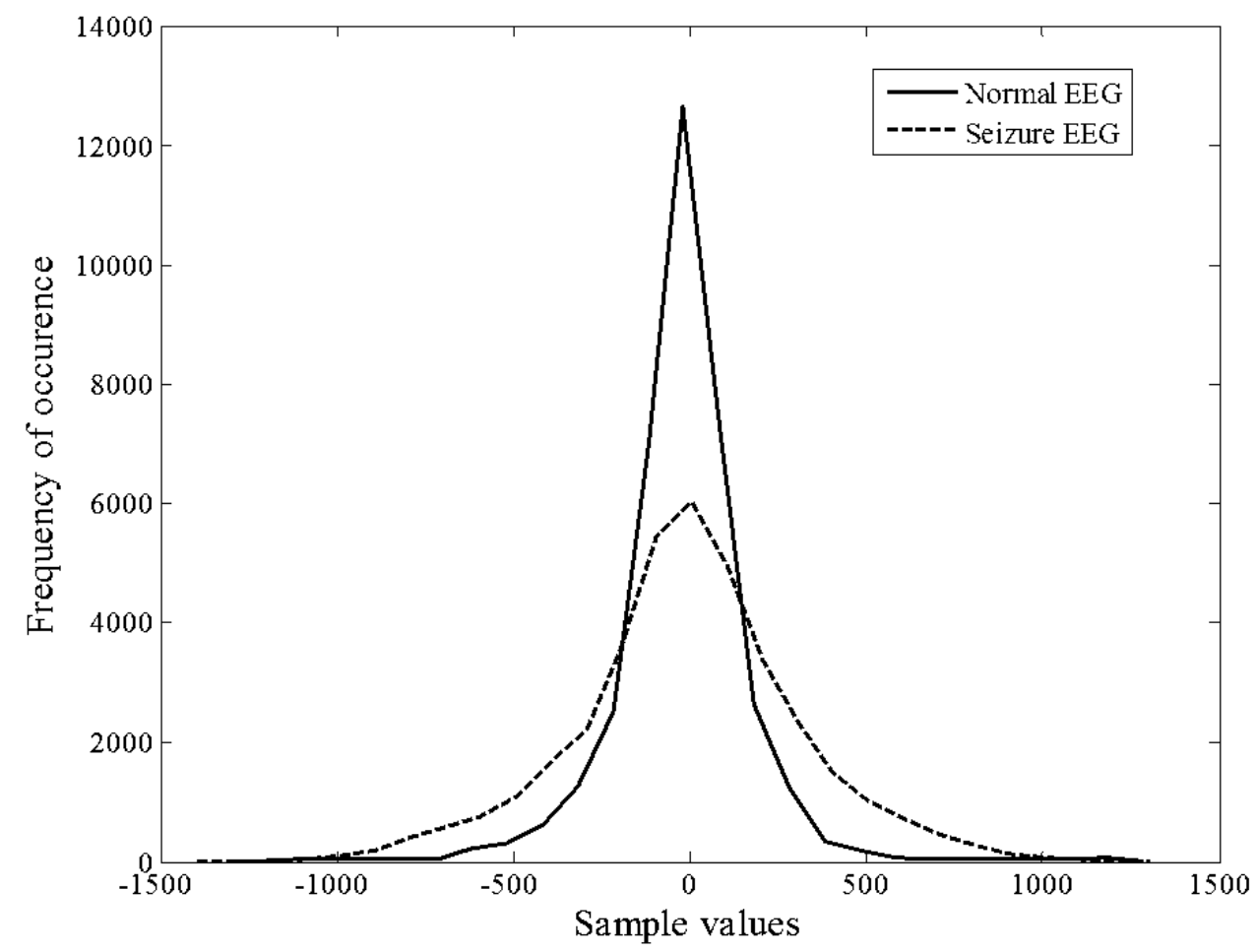

Figure 1: Histogram of seizure and normal EEG

The seizure data was first divided into frames of 1 second each using non-overlapping epoch window. A background window of 25 seconds was taken to normalize the epoch features and this window was made to move with epoch window (Figure 2a and Figure 2b). A gap of 15 seconds between epoch \& background was taken to prevent seizure onset into the background (Figure 2a).

The background window was also divided into frames of 1 second and then each epoch of seizure and frames of background window were decomposed up to level 5 using Daubechies wavelet of order 4. Since most of the seizure information lies in $0.5-30 \mathrm{~Hz}$ range, levels A5 (0-4

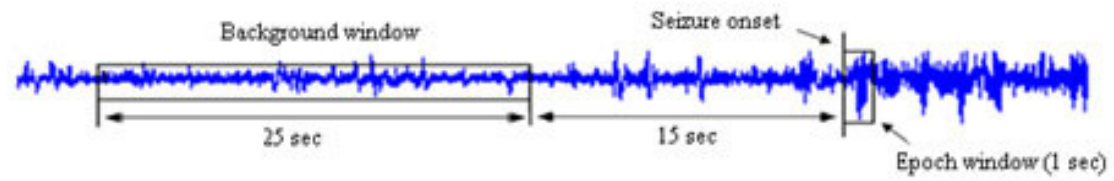

Figure 2a. A segment of EEG showing seizure onset, background window and epoch window 
International Journal of Embedded Systems and Applications (IJESA) Vol.2, No.3, September 2012

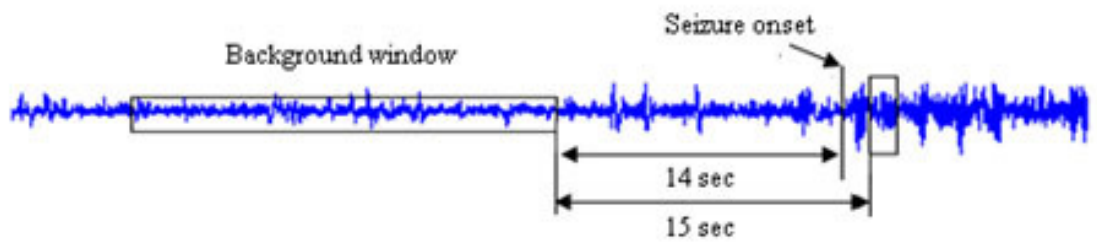

Figure $2 \mathrm{~b}$. A segment of EEG, showing movement of background window

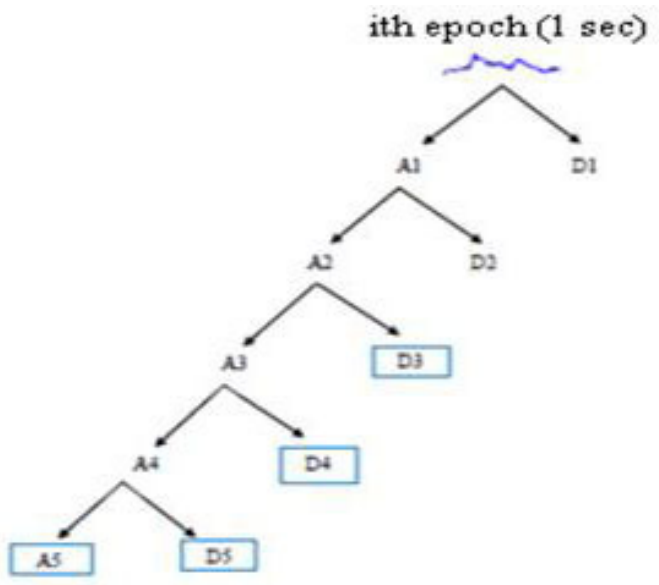

Figure 3. Five level wavelet decomposition

$\mathrm{Hz}), \mathrm{D} 5(4-8 \mathrm{~Hz}), \mathrm{D} 4(8-16 \mathrm{~Hz})$ and D3 $(16-32 \mathrm{~Hz})$ were used for the computation of features. The used wavelet levels are shown in blue boxes in Figure 3.

NCOV for each epoch was computed using equation (1)

$$
\mathrm{NCOV}=\frac{\mathrm{NCOV}_{\mathrm{e}}}{\mathrm{NCOV}_{\mathrm{b}}}
$$

where, NCOVe is normalized coefficient of variation for seizure epoch and NCOVb is average value of normalized coefficient of variation for background window. These can be calculated using equations (2) \& (3) respectively.

$$
\begin{aligned}
\operatorname{NCOV}_{\mathrm{e}} & =\frac{\sigma_{\mathrm{x}}^{2}}{\mu_{|\mathrm{x}|}} \\
w_{k} & =\frac{\sigma_{\mathrm{x}_{\mathrm{k}}}^{2}}{\mu_{\left|\mathrm{x}_{\mathrm{k}}\right|}} \\
\operatorname{NCOV}_{\mathrm{b}} & =\operatorname{mean}\left(\mathrm{w}_{\mathrm{k}}\right), \mathrm{k}=1 \text { to } 25
\end{aligned}
$$

where, $\mathrm{x}$ is the sample value 
International Journal of Embedded Systems and Applications (IJESA) Vol.2, No.3, September 2012

Kurtosis and skewness were the statistical features computed over the raw EEG. Both are the representative of the shape of the probability distribution of the data. Skewness is a measure of asymmetry while kurtosis is a measure of peakedness [15]. Consequently total 6 features were extracted for each epoch on a channel which is shown in Figure 4.

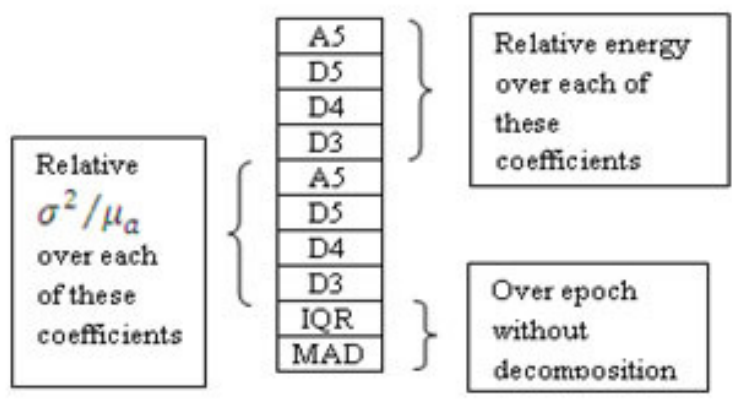

Figure 4. Features used in this study

Mostly 23 channels were used for recording in database. So for each epoch a vector of $23 * 6$ dimensions was formed and because a seizure is of different duration (T), T such epochs would be there. A feature vector was formed by concatenating $23 * 6$ dimension vector of each epoch vertically. Hence the dimension of feature vector was $(23 * \mathrm{~T}) * 6$. This feature vector is for seizure EEG signal. Similarly feature vectors for normal EEG signal were calculated. Normal EEG signal of $\mathrm{T}$ sec duration was taken randomly from non-seizure records. It was assumed that normal data, used for feature vector formation, was free from artifacts. Since, 55 seizures were present in the first 10 patient's EEG of database, 55 feature vectors were formed for seizure and 55 feature vectors were similarly formed for normal EEG signals.

\subsection{Results and Discussion}

Classification between the normal and seizure EEG signal was done by inputting the extracted features to the linear classifier. These are of discriminative type i.e. they learn the way of discriminating the classes in order to classify a feature vector. It uses hyper-planes to separate the data representing different classes. If the problem is a two class problem such as seizure and nonseizure type, the class of feature vector depends on which side of hyper plane it lays. The separating hyper plane is that plane for which the distance between two classes' means is maximum and interclass variance is minimum [16]. Here the classification was done for each patient separately and the results obtained were averaged out to get the final result. For example patient 6 was having 10 seizures, so 8 of them were used for training and 2 were used for testing at a time and this process was repeated until every seizure got tested.

The classification was done to differentiate between two classes: seizure and normal EEG. Seizure epoch was labelled using 1 and normal epoch was labelled using 0 . The classifier declared the seizure in any epoch if it was present in at least $60 \%$ channels. This was done to eliminate the artifact detection as seizures.

The performance of the classification was measured using the metrics: latency, sensitivity and false detection rate. Latency is the term used for the delay between the expert marked seizure onset and the detected seizure onset. Sensitivity refers to the number of seizures detected. False 
International Journal of Embedded Systems and Applications (IJESA) Vol.2, No.3, September 2012

detection rate refers to the number of times the detector declared the seizure during the course of 1 hour when it was not present actually.

The mean latency with which the seizure declared the onset of every seizure was 3.2 seconds. Figure 5 is showing the mean latency and Figure 6 is showing the average number of false detections per hour of each of the 10 patients.

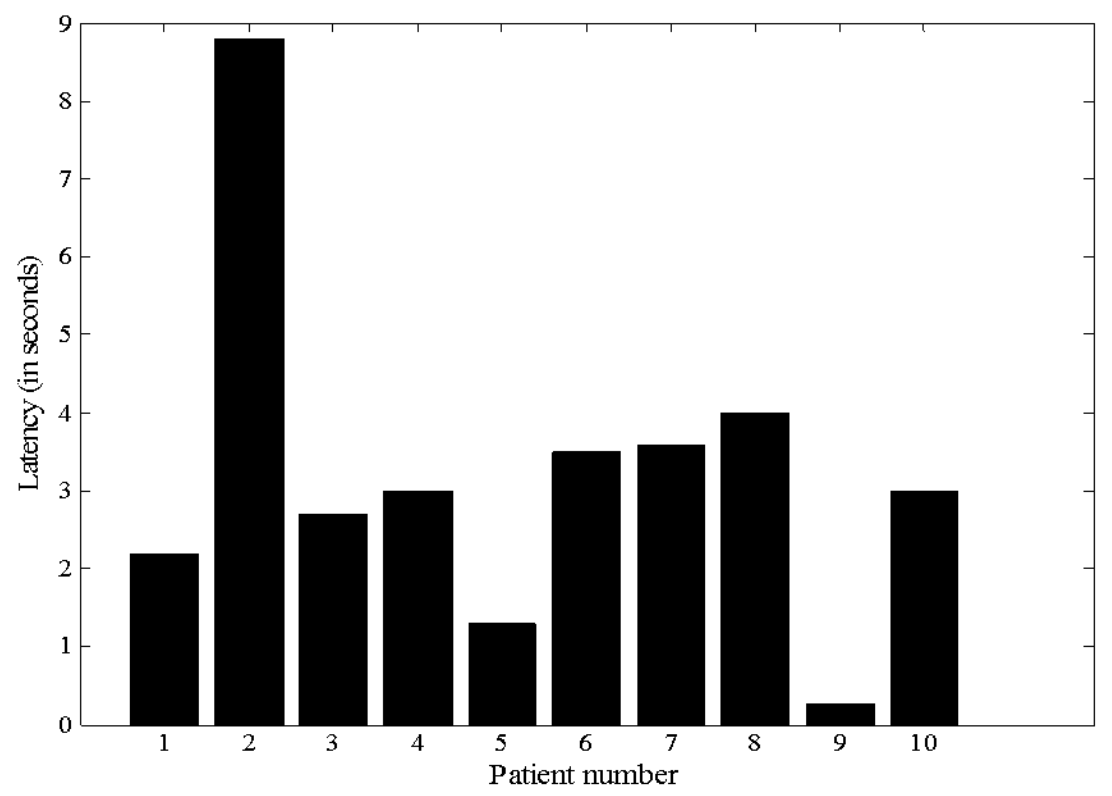

Figure 5. Average latency for each patient

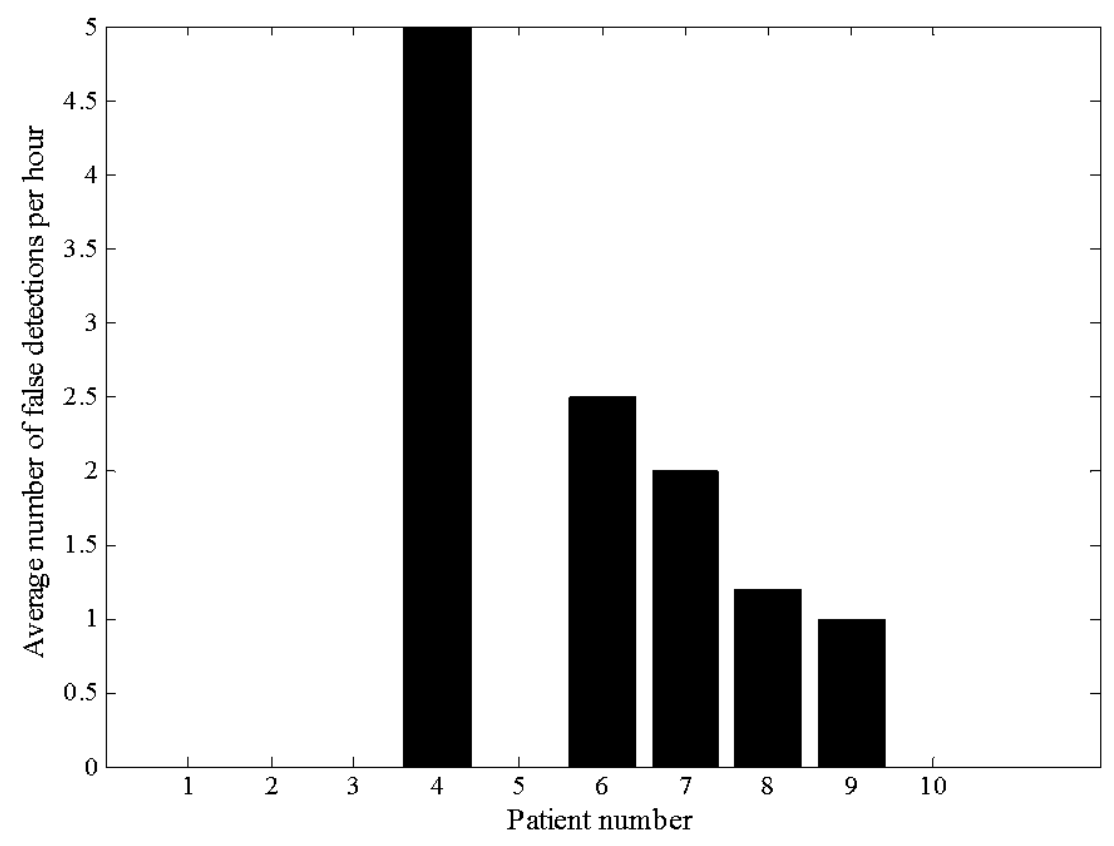

Figure 6. Average false detections per hour for each patient 
International Journal of Embedded Systems and Applications (IJESA) Vol.2, No.3, September 2012

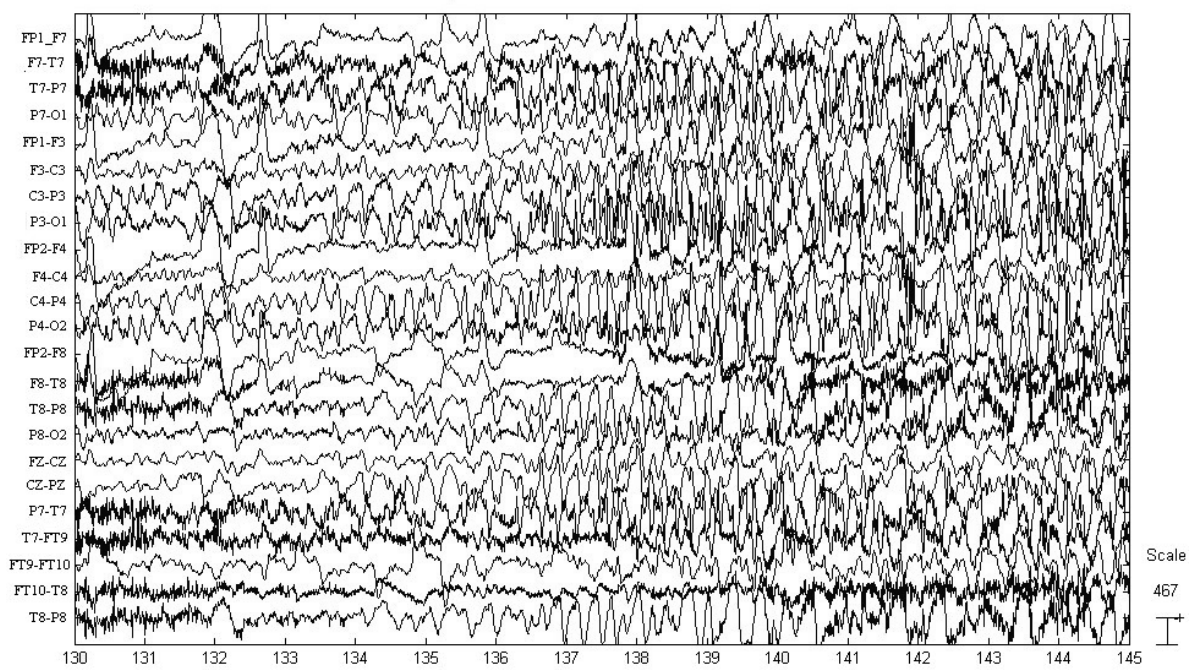

Figure 5a. 15 seconds' EEG section of one of the seizures present in patient2

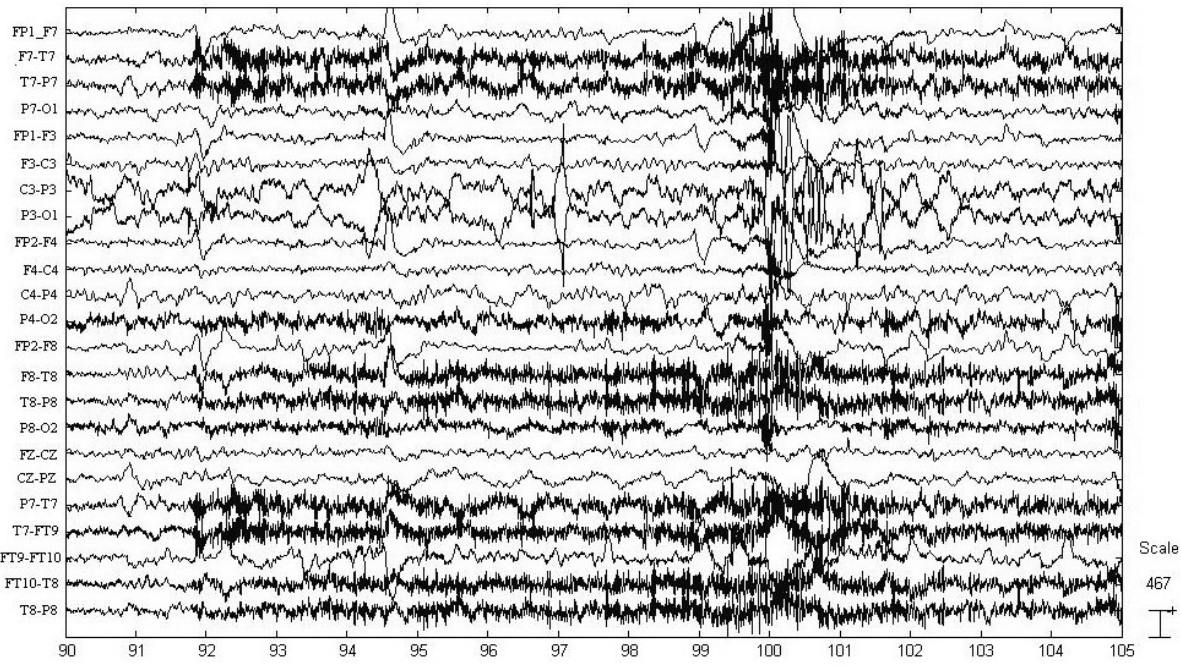

Figure 5b. Background EEG taken to normalize the seizure shown in Figure 5a.

The maximum latency in seizure onset detection was observed in patient 2. Figure 6a and Figure $6 \mathrm{~b}$ shows $10 \mathrm{sec}$ EEG of one of the seizures present in patient 2 and background window taken for normalizing it respectively. The detector delays in detecting the onset of this seizure since its starting high amplitude characteristics are similar to background EEG characteristics. The maximum number of false detections was observed in case of patient 4 . This is due to the artifact full EEG of this patient. The reason of getting zero false detection in $50 \%$ of the patients is the clean normal taken for classification.

Since the detector detected the onset of every seizure used for this study, hence the sensitivity achieved was $100 \%$. The average number of false detections observed by the detector was 1.1 per hour. 
International Journal of Embedded Systems and Applications (IJESA) Vol.2, No.3, September 2012

\section{Conclusions}

In this work, statistical features in combination with wavelet based features were extracted and a method to detect the onset of seizures with low latency has been proposed. The onset was detected only if the seizure is present on more than $60 \%$ of the channels. The methodology was able to detect all the seizures (55 in 10 patients) with the average latency of 3.2 seconds. In future, more features with additional discriminatory information will be investigated to further improve the results.

\section{REFERENCES}

[1] A. Dorai, and K. Ponnambalam, "Automated epileptic seizure onset detection”, in Autonomous and Intelligent Systems (AIS), 2010 International Conference on, Povoa de Varzim, 21-23 June, 2010, pp. $1-4$.

[2] T. L. Sorensen, U. L. Olsen, I. Conradsen, J. Henriksen, T. W. Kjaer, C. E. Thomsen, and H. B. D. Sorensen, "Automatic epileptic seizure onset detection using Matching Pursuit: A case study", in Engineering in Medicine and Biology Society (EMBC), 2010 Annual International Conference of the IEEE, 2010, pp. 3277-3280.

[3] I. Yaylali, H. Kocak, and P. Jayakar, "Detection of seizures from small samples using nonlinear dynamic system theory”, IEEE Trans. on Biomed. Engg., vol. 43, no. 7, pp. 743-751, July, 1996.

[4] G. Geetha, and S. N. Geethalakshmi, "Detecting Epileptic Seizures Using Electroencephalogram: A New and Optimized Method for Seizure Classification using Hybrid Extreme Learning Machine", in Process Automation, Control and Computing (PACC), 2011 International Conference on, Coimbatore, 20-22 July 2011, pp. 1-6.

[5] J. Gotman, and P. Gloor, "Automatic recognition and quantification of interictal epileptic activity in the human scalp EEG”, Electroencephalography and Clinical Neurophysiology, vol. 41, pp. 513-529, 1976.

[6] J. Gotman, "Automatic recognition of epileptic seizures in the EEG", Electroencephalography and Clinical Neurophysiology, vol. 54, no. 5, pp. 530-540, 1982.

[7] H. Qu, and J. Gotman, "A patient-specific algorithm for the detection of seizure onset in long-term EEG monitoring: Possible use as a warning device”, IEEE Trans. on Biomed. Engg., vol. 44, no. 2, pp. 115-122, Feb 1997.

[8] M. E. Saab, and J. Gotman, “A system to detect the onset of epileptic seizures in scalp EEG”, Clinical Neurophysiology, vol. 16, no. 2, pp. 427-442, 2005.

[9] A. Shoeb, and J. Guttag, "Application of Machine Learning To Epileptic Seizure Detection”, in Proceedings of the 27th International Conference on Machine Learning, Haifa, Israel, 2010, pp. 975982.

[10] CHB-MIT scalp EEG database [Online]. Available: http://physionet.org/physiobank/database/chbmit/

[11] A. Kharbouch, A. Shoeb, J. Guttag, and S. S. Cash, "An algorithm for seizure onset detection using intracranial EEG”, Epilepsy Behav., vol. 22, pp. S29-35, 2011.

[12] H. Adeli, Z. Zhou, and N. Dadmehr, "Analysis of EEG records in an epileptic patient using wavelet transform,” J. Neurosci. Methods, vol. 123, no. 1, pp. 69-87, 2003. 
International Journal of Embedded Systems and Applications (IJESA) Vol.2, No.3, September 2012

[13] T. Fathima, M. Bedeeuzzaman, O. Farooq, and Y. U. Khan, "Wavelet Based Features for Epileptic Seizure Detection”, MES Journal of Technology and Management, vol. 2, no. 1, pp. 108-112, May 2011.

[14] Y. U. Khan and O. Farooq, "Autoregressive features based classification for seizure detection using neural network in scalp Electroencephalogram”, Int. J. Biomed. Engg. Technology, vol. 2, no. 4, pp. 370-381, 2009.

[15] M. Bedeeuzzaman, O. Farooq, Y. U. Khan, "Dispersion measures and entropy for seizure detection", Acoustics, Speech and Signal Processing (ICASSP), 2011 IEEE International Conference on , Prague, 22-27 May 2011, pp.673-676.

[16] F. Lotte, M. Congedo, A. L'ecuyer, F. Lamarche, and B. Arnaldi, "A review of classification algorithms for EEG-based brain-computer interfaces”, J. Neural Eng., vol. 4, pp. R1-13, 2007.

\section{Authors}

Yusuf Uzzaman Khan did his B. Sc. in Engineering from A.M.U. in 1990 and M.E. in 1993 from University of Roorkee, India. He was a Felix scholar for D.Phil. in Oxford University from 1994 to 1997. He is an Associate Professor in the Electrical Engineering Department of A.M.U. Aligarh. His research interests are in biomedical signal processing, wavelets and neural networks. He has wide publications in the conferences and refereed journals.

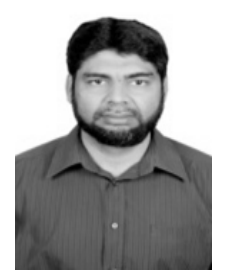

Omar Farooq obtained B. Sc. Engineering and M. Sc. in Engineering from Z.H. College of Engineering and Technology, AMU, in 1991 and 1993, respectively. He joined the Department of Electronics Engineering as a lecturer in 1992 and promoted to reader in 2002. He obtained his $\mathrm{PhD}$ from Loughborough University, UK, under the Commonwealth scholarship in2002 carried out one year post-doctoral research under UKIERI scheme in 2007-2008. His area of research is signal processing and pattern recognition.

Priyanka Sharma is pursuing M.Tech in Department of Electronics Engineering (II year) from ZHCET, AMU, Aligarh, India. This work has been carried out during her dissertation in Biomedical Research Division, ZHCET, AMU, Aligarh, India. She did the work on the detection of convulsive seizures and the work is going to be published in the conference proceedings of Springer.
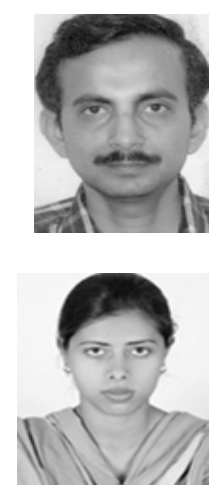